\author{
I. Köster · L. von Ferber · P. Ihle · I. Schubert • \\ H. Hauner
}

\title{
The cost burden of diabetes mellitus: the evidence from Germany_the CoDiM Study
}

Received: 9 November 2005 / Accepted: 13 March 2006 / Published online: 11 May 2006

C) Springer-Verlag 2006

\begin{abstract}
Aims/hypothesis: The aim of this study was to identify the health care costs of diabetic patients in Germany in 2001, focusing on the influence of age, sex, and type of treatment. Subjects and methods: Annual direct costs of medical care and indirect costs of inability to work and early retirement in diabetic subjects were compared with costs of age- and sex-matched non-diabetic control subjects. The analysis was based on routine health care data from a random sample (18.75\%) taken from a database of 1.9 million insured persons. Incremental differences in medical and national expenditure between subjects with and without diabetes were calculated. Results: Annual direct mean costs per diabetic patient were $€ 5,262$, and indirect costs were $€ 5,019$. In the control group, mean direct and indirect costs were $€ 2,755$ and $€ 3,691$, respectively. Analysis of cost components revealed that the high costs associated with the care of diabetic patients could be largely attributed to inpatient care and overall medication costs. Hypoglycaemic drugs amounted to only one-quarter of the medication costs. The total health care costs were correlated with the type of treatment. Direct excess costs increased with increasing age in insulin-treated patients, but were unaffected by age in patients receiving other types of treatment. Conclusions/interpretation: The Costs of Diabetes Mellitus (CoDiM) study is the first comprehensive study to
\end{abstract}

Electronic Supplementary Material Supplementary material is available for this article at http://dx.doi.org/10.1007/s00125-0060277-5

I. Köster $(\bowtie) \cdot$ L. von Ferber $\cdot$ P. Ihle $\cdot$ I. Schubert

PMV Research Group, University of Cologne,

Herderstrasse 52-54,

50931 Cologne, Germany

e-mail: ingrid.koester@uk-koeln.de

Tel.: +49-221-4786545

Fax: +49-221-4786766

H. Hauner

Else Kröner Fresenius Centre for Nutritional Medicine

Munich Technical University,

Munich, Germany provide estimates of costs associated with diabetes care in Germany. Direct costs of diabetic patients account for $14.2 \%$ of total health care costs, which includes the proportion that specifically accounts for diabetes-related costs $(6.8 \%)$.

Keywords Complications - Costs - Diabetes mellitus Germany $\cdot$ Hypoglycaemic drugs $\cdot$ Medical care

Abbreviations ADA: American Diabetes Association ATC: Anatomical-Therapeutic-Chemical classification . CoDiM: Costs of Diabetes Mellitus - ESM: Electronic supplementary material - ICD: International Classification of Diseases $\cdot \mathrm{KV}$ : Association of Statutory Health Insurance Physicians · SGB: Social Security Code

\section{Introduction}

Diabetes mellitus is a public health issue of significant economic importance because of the chronic nature of diabetes, its high and globally increasing prevalence, the demand for multi-modal treatment, and the serious complications associated with long disease duration. Data from the United States and Europe indicate that the health care expenditure for patients with diabetes mellitus is significantly higher than for comparable patients without this disease [1-5]. These and other studies [6-9] also suggest that the majority of such 'excess' costs can be attributed to diabetes-related complications.

Cost estimates for diabetes derived from different studies are difficult to compare because of methodological and other differences in cost appraisal [2]. Furthermore, most study data are derived from aggregated data sets and their validity and reliability are consequently uncertain. Therefore, the use of alternative data sets and methodology may be required to obtain a definitive insight into the costs of diabetes as they affect the health care system and society. There is a need for studies that examine the impact of individual variables, such as type of treatment and patient age and sex, on health care expenditures. 
In Germany, $86 \%$ of the population is fully covered by the social security system consisting of statutory health insurance funds. Insured persons have free choice among different health insurance funds. For this Costs of Diabetes Mellitus (CoDiM) study, we used the database of the largest statutory health insurance company in Germany (AOK), which insures approximately 26.5 million peopleone-third of the German population. The data from a random sample were used for a comprehensive and detailed analysis of the health care costs per diabetic patient and of the influence of patient age, sex, and type of treatment on these costs. The cost to society of lost wages and early retirement are included as indirect costs. In addition, we determined the excess costs associated with diabetes through a comparison with an age- and sexmatched non-diabetic control population.

\section{Subjects and methods}

The CoDiM study is based on routine health care data collected in 2001 by AOK, the local statutory health insurance fund, and $\mathrm{KV}$, the Association of Statutory Health Insurance Physicians, of the Federal State of Hesse in Germany. The Federal State of Hesse has a population of 5.8 million, of which 1.9 million people receive health insurance through AOK.

A random sample of continuously insured persons in the AOK Hesse database in $2001(n=306,736)$ was taken. For each insured individual, it was possible to generate patientrelated histories of treatment by record linkage, including outpatient care, inpatient care, services of nursing insurance, and days off work due to illness. The database also included information relating to the extent and cost of treatment, as well as sociodemographic variables, such as patient age, sex, period of insurance coverage, and insurance status (member, pensioner, co-insured dependent). The anonymity of the randomly selected patient insurance data retrieved was protected by the use of pseudonyms.

Identification of subjects with diabetes

An insured person was identified as a diabetic patient if at least one of the following criteria was fulfilled [10]: (1) regular documentation of 10th International Classification of Diseases (ICD-10) diagnoses 'diabetes' (E10-E14) in three of the four quarters of the year 2001; (2) regular prescription of hypoglycaemic drugs (Anatomical-Therapeutic-Chemical [ATC] classification A10); at least two prescriptions in 2001; (3) a single prescription of hypoglycaemic drugs in 2001 plus a diagnosis of 'diabetes' or blood glucose or $\mathrm{HbA}_{1 \mathrm{c}}$ measurement in the same quarter (to avoid false-positive cases due to data errors). Patients without prescription of hypoglycaemic drugs and with fewer diabetes diagnoses were classified as suspicious cases and were excluded from the analyses. Classification of diabetic subjects according to type 1 or type 2 disease was not possible because a large number of physicians coded the diagnoses of diabetes in the ICD-10 class E14, which corresponds to the general diagnosis of diabetes mellitus.

\section{Matched case-control}

Following the selection of diabetic patients from persons insured with AOK in Hesse throughout 2001, each diabetic patient was matched by age and sex with a non-diabetic control selected randomly from the same database. Nondiabetic controls were identified as persons without any ICD-10 diagnosis of 'diabetes' and without any prescription of hypoglycaemic drugs in 2001.

\section{Cost analysis}

Direct and indirect costs for 2001 were calculated for each diabetic patient and control. Excess costs were defined as the difference in average costs of diabetic patients and the control group. Direct costs of diabetes included (1) services provided via health insurance (based on Social Security Codes [SGB] V [11]), specifically, inpatient care, medications, physicians' outpatient services, medical devices (e.g. glasses, wheelchairs, etc.), home care, transportation (transportation of sick persons, which stands in relation to a medical service provided by the sickness fund, necessary for medical reasons [SGB V \$60], e.g. transportation of a handicapped patient to a medical doctor, transportation in an ambulance vehicle), other remedies (e.g. physiotherapy); and (2) services provided via nursing insurance (SGB XI [11]), such as long-term nursing care. Indirect costs included loss of productivity as a result of the inability to work or early retirement.

Data of physicians' outpatient care were taken from KV, the association of statutory insurance physicians, of the Federal State of Hesse in Germany. The costs were calculated by multiplying the quantities of the resource used by the unit price of each resource taken from the Einheitlicher Bewertungsmaßstab (2001 values [12]). The prices and charges of all other outpatient services (medication, medical devices, care of home, transportation, remedies), inpatient care and long-term nursing care were based on reimbursement fees taken from the AOK database.

The AOK also documents data on inability to work as a result of illness (SGB V \$44-51). The number of days off work because of illness was identified by these data. Early retirement was identified by the insurance status 'pensioner' before the actuarial age of entry to a pension (persons younger than 65 years). Indirect costs were calculated by multiplying the number of days of worker absenteeism (days off work because of illness or days of early retirement) with a sex-specific average income ( $€ 2,984$ for men and $€ 2,340$ for women per month), based on data from the Federal Statistical Office (October 2001) [13].

The costs of diabetes were categorised as follows: (1) management of the primary disease (i.e. hyperglycaemia); 
(2) management of diabetes-related disorders (i.e. comorbidities and complications [excess costs minus costs of management of diabetes]); and (3) management of disorders unrelated to diabetes (i.e. costs of the controls).

Costs of management of diabetes included hypoglycaemic drugs, devices for insulin delivery and blood glucose self-monitoring, blood glucose and $\mathrm{HbA}_{1 \mathrm{c}}$ measurement, patient education and counselling (such as that provided by doctors and dietitians), and inpatient care necessitated by abnormal blood sugar levels (e.g. ketoacidosis, excluding diabetic complications).

\section{Statistical analysis}

Estimates of prevalence rates and average costs per patient are provided. We used 2.5 and 97.5 percentiles and SD as measures of variation. Data were standardised according to the age and sex distribution of the German population (82.3 million inhabitants on 31 December 2000). Costs were projected on the basis of estimated age- and sex-specific prevalence rates and per-patient costs in the German population. The influences of age, sex and type of therapy on the annual direct costs were calculated by multiple linear regression (costs were log transformed). All analyses were performed using SAS Windows Version 8.2 (SAS Institute, Cary, NC, USA).

\section{Results}

Characteristics of the diabetic cohort

A total of 26,971 subjects in the AOK database (2001) were identified as diabetic patients. This represents a prevalence of $6.9 \%(6.5 \%$ male; $7.4 \%$ female $)$ when standardised to represent the German population at the time of the study. The estimated number of diabetic patients in Germany in 2001 is 5.818 million. Of the diabetic patients, 0.957 million (prevalence $1.1 \%$ ) were exclusively treated with insulin; 0.651 million (prevalence $0.8 \%$ ) were treated with insulin and oral agents (combined insulin/oral agents or change in therapy in 2001); 2.581 million (prevalence $3.1 \%$ ) received oral agents alone; and 1.629 million (prevalence $1.9 \%$ ) were not prescribed any hypoglycaemic drugs.

Health-resource utilisation attributable to diabetes

In 2001, diabetic patients had a mean of 31.3 contacts with outpatient general practitioners or specialists compared with 19.9 such contacts in the control group. Diabetic patients were also more likely to visit different physicians than controls (mean 4.2 vs 3.5 physicians per year). In $2001,28.8 \%$ of the diabetic population had at least one

Table 1 Division of the annual costs $(€)$ per person according to cost components in the CoDiM sample in 2001

\begin{tabular}{|c|c|c|c|c|c|c|}
\hline \multirow[t]{3}{*}{ Included costs } & \multicolumn{3}{|c|}{ Costs per person $(€)$} & \multicolumn{3}{|c|}{ Diabetes excess costs $(€)$} \\
\hline & \multicolumn{2}{|c|}{ Diabetic patients } & \multirow{2}{*}{$\begin{array}{l}\text { Control } \\
\text { subjects } \\
\text { Mean } \\
\text { (C) }\end{array}$} & \multirow{2}{*}{$\begin{array}{l}\text { Ratio of diabetic } \\
\text { patients to controls } \\
(D / C)\end{array}$} & \multirow{2}{*}{$\begin{array}{l}\text { Total diabetes } \\
\text { excess costs } \\
(D-C)\end{array}$} & \multirow{2}{*}{$\begin{array}{l}\text { Excess costs for } \\
\text { management of the primary } \\
\text { disease }^{\mathrm{a}}\end{array}$} \\
\hline & $\begin{array}{l}\text { Mean } \\
(D)\end{array}$ & $\begin{array}{l}2.5-97.5 \\
\text { percentile }\end{array}$ & & & & \\
\hline Inpatient care & 1,870 & $0-16,259$ & 1,024 & 1.8 & 846 & 52 \\
\hline Medications & 1,006 & $39-3,599$ & 445 & 2.3 & 561 & 251 \\
\hline Physicians' outpatient services & 778 & $104-2,278$ & 467 & 1.7 & 311 & 124 \\
\hline Medical devices & 372 & $0-2,510$ & 143 & 2.6 & 229 & 115 \\
\hline Care at home & 111 & $0-1,793$ & 23 & 4.8 & 88 & - \\
\hline Indirect costs & 5,019 & $0-35,808$ & 3,691 & 1.4 & 1,328 & - \\
\hline Inability to work & 764 & $0-7,385$ & 576 & 1.3 & 188 & - \\
\hline Early retirement & 4,255 & $0-35,808$ & 3,115 & 1.4 & 1,140 & - \\
\hline Total & 10,281 & $312-44,303$ & 6,446 & 1.6 & 3,835 & 542 \\
\hline
\end{tabular}

All values (except percentiles) have been standardised according to the age and sex distribution of the German population ( 82.3 million inhabitants at 31 December 2000)

${ }^{a}$ Cost components identifiable 
hospital admission compared with $19.1 \%$ of the control group. On average, diabetic patients required a mean of 29.9 drug prescriptions per year compared with 15.9 prescriptions per year in the control group.

\section{Annual costs of diabetes}

Table 1 shows the average annual costs per diabetic patient when standardised to represent the German population at this time; mean direct costs were $€ 5,262$ (unstandardised: $€ 5,519 \pm 8,896$ ), while indirect costs were $€ 5,019$ (unstandardised: $€ 4,609 \pm 10,890)$. In the control group, average direct and indirect costs were $€ 2,755$ and $€ 3,691$, respectively (unstandardised: $€ 2,993 \pm 5,914$ and $€ 3,465 \pm$ 9,566). Direct and indirect excess costs as a result of diabetes were estimated at $€ 2,507$ and $€ 1,328$, respectively.

Hence, the total direct costs of diabetic subjects were 1.9 times those of non-diabetic subjects. According to the specific cost components this ratio varied from a high of 4.8 for care at home to a low of 1.5 for other remedies. Direct excess costs were compiled from costs of medical care (sick fund) $€ 2,193$ (87.5\%) and long-term nursing care (nursing insurance) $€ 314(12.5 \%)$. Analysis of specific cost components revealed that the excess costs could be largely attributed to inpatient care $(33.7 \%)$ and overall medication $(22.4 \%)$, with $10.0 \%$ of direct excess costs being attributable to hypoglycaemic drugs $(€ 251$ per patient per year). Physicians' outpatient services accounted for $12.4 \%$ of the medical care expenditure in the diabetic cohort, representing the third highest direct excess cost at $€ 311$ per patient.

Direct costs of diabetes according to type of treatment, age and sex

The log-transformed annual direct costs fitted by multiple regression to the indicator variables sex, age, and type of therapy accounted for $21.2 \%$ of the variance in costs. All variables were highly significant $(p<0.001)$. Significant interactions were found between sex and age and between type of therapy and age $(p<0.001)$; no significant interaction was found between type of therapy and sex.

Type of diabetes treatment had a strong impact on direct costs. Patients on insulin monotherapy or insulin and oral hypoglycaemic drugs had substantially higher costs compared with the control group (ratios of 3.4 and 3.1, respectively), whereas patients on oral hypoglycaemic drugs alone and patients managed by diet and exercise alone exhibited only moderately elevated costs (ratio of 1.4; see Electronic supplementary material [ESM] Table 1).

Age was a strong determinant of direct expenditure in all subjects and a parallel increase in costs with age occurred in all subgroups. The ratio of direct costs in diabetic subjects to such costs in non-diabetic subjects was 4.1 in subjects aged $<40$ years, but decreased to 1.5 in those aged $\geq 80$ years. This feature was consistent across all treatment groups.
In patients receiving oral hypoglycaemic drugs and in unmedicated patients, the direct excess costs of diabetes were unaffected by age. These data contrast with the agedependent increase in excess costs in patients on insulin therapy. Insulin-treated patients aged $\geq 80$ years had mean excess costs of $€ 7,839$ compared with mean excess costs of $€ 3,859$ in patients aged $<40$ years. A similar observation was made in patients treated with insulin and oral hypoglycaemic drugs. For all diabetic patients, excess costs decreased slightly up to the 50- to 59-year age group and increased slightly thereafter. This trend mirrored the structure of the individual age groups according to type of therapy: at most, $61 \%$ of young diabetic patients $(<40$ years) received insulin therapy ( \pm oral hypoglycaemic drugs); this decreased to $30 \%$ in the 40 - to 49 -year age group, and still further to $23-27 \%$ in older patients.

The increase in the excess costs of diabetes with advancing age in patients on insulin alone and in combination with oral hypoglycaemic drugs was not the result of increases in the cost of management of the primary disease (i.e. hyperglycaemia), but of expenditures for the treatment of diabetes-related co-morbidities and complications. Indeed, the basal costs of treating diabetes decreased with increasing patient age; in particular, the cost of medical devices (including tools for insulin delivery and the self-monitoring of blood sugar) decreased. Also the cost of hypoglycaemic drugs in patients aged $\geq 70$ years decreased.

\section{Direct costs of diabetes in Germany}

In projecting the prevalence and annual direct costs per diabetic patient in the German population in 2001, direct costs of diabetes were estimated to be $€ 30,616$ million, including excess costs of $€ 14,584$ million $(100 \%)$, of which $€ 4,922$ million $(33.7 \%)$ was for inpatient care; $€ 3,264$ million (22.4\%) was for overall medications; $€ 1,808$ million $(12.5 \%)$ was for physicians' outpatient services; and $€ 2,256$ million (15.4\%) was for medical devices, other remedies, transportation, and other outpatient services. $€ 509$ million (3.5\%) was for care at home and $€ 1,826$ million (12.5\%) was for long-term nursing care.

By sex, $44.8 \%$ of the total direct excess costs were for the treatment of men and $55.2 \%$ were for women. By age, $4.4 \%$ of total medical expenditures were for diabetic patients $<40$ years, $51.3 \%$ were for those $40-69$ years, and $44.3 \%$ were for those $>69$ years. By type of diabetes therapy, $€ 9,633$ million $(66.1 \%)$ of total direct excess costs were for diabetic patients on insulin either alone or in combination with oral hypoglycaemic drugs.

\section{Discussion}

This prevalence-based cost-of-illness study involved a large population of patients with diabetes mellitus from the Hesse region in Germany. The analysis was based on cost 
and administrative data retrieved from an insurance company database for 2001. The CoDiM study is the first study to provide estimates of the distribution of costs associated with diabetes care. Such an analysis is only possible when a 'bottom-up' approach is taken.

According to the Organization for Economic Cooperation and Development, the total health care expenditure in Germany for the year 2000 was $€ 215$ billion [14]. The results of the CoDiM study reveal that in Germany the direct costs of diabetic patients account for $€ 30.6$ billion $(14.2 \%)$ of total health care expenditure, including an expenditure of $€ 14.6$ billion (6.8\% of total) directly due to diabetes.

Cost estimates from the CoDiM study were clearly higher than those provided for Germany by the Costs of Diabetes in Europe-type 2 study (CODE-2), a 1-year, bottom-up, prevalence-based study, which compared the costs of treatment of type 2 diabetes in eight European countries $[3,4,15]$. Variation in these estimates of diabetes costs are caused by different methods of patient recruitment, differences in the data survey, and inherent differences between study years.

The cost estimates of the CoDiM study were lower than those reported for studies in the USA [9, 16, 17] irrespective of the specific cost components studied. Notably, hospital costs recorded by the US American Diabetes Association (ADA) study [16] were much higher than those obtained by the CoDiM study or the mean costs of the European CODE-2 study [4]. In retrospect, these cost differences may be attributed to high US hospital expenditures, which are significantly greater than in Europe. For example, US hospital expenditures in each case (1999) were 2.7-fold greater than those recorded in Germany [18].

The health care cost ratios, indicative of excess costs, for diabetic versus non-diabetic subjects have been estimated in various cost-of-illness studies in the USA, Canada and Sweden $[1,9,16,17,19,20]$. Cost ratios varied greatly between studies, ranging from 2.1 to 5.2. In the CoDiM study, the cost ratio (based on direct costs) between diabetic and non-diabetic subjects was 1.9. First, the cost ratio is dependent on the characteristics of the non-diabetic cohort. For example, adjustments in the ADA study for demographic characteristics between cohorts, such as sex, age, race, ethnic group, etc, that are potentially correlated with the use of health care resources decreased the cost ratio from 5.2 to 2.4 [16]. The cost ratio is strongly dependent on cohort age structure and type of diabetes therapy. When adjusted for type of therapy and age of the diabetic cohort, the cost ratio in the CoDiM study ranged from 1.2 (in elderly diabetic patients not on insulin therapy) to 4.9 (in young diabetic patients on insulin therapy). Correspondingly, US studies place the cost ratio of elderly diabetic patients well below that of young diabetic patients [1]. In a Swedish study, which included only young or middle-aged subjects, the cost ratio was 4.8 [21].

Until now, few cost-of-diabetes studies have stratified per-patient costs according to age $[1,9,22,23]$ and type of therapy $[4,23,24]$. Indeed, these characteristics have typically been assessed by univariate analysis. Using multiple linear regression analysis, one study group investigated the effect of age and type of therapy, as well as other variables, on the direct costs of diabetes [6]. However, correlations between age group and type of therapy were not included in this model.

The direct costs for diabetic patients receiving insulin therapy in the CoDiM study were twice those of diabetic patients receiving other or no medications; only slight differences in cost were found between patients receiving oral hypoglycaemic drugs and unmedicated patients. Similar conclusions have been drawn by other studies comparing the cost of therapy in diabetes [23, 24].

The CoDiM study shows that, in addition to the higher costs of management of the primary disease (i.e. hyperglycaemia), patients on insulin therapy have significantly higher costs for the treatment of the complications and diseases associated with diabetes and that these excess costs increase with patient age. Costs were lower in unmedicated diabetic patients or in those treated with oral hypoglycaemic drugs, with only a slight difference between age groups (see ESM Table 1).

The trend of increased excess cost with age found in patients on insulin therapy did not persist when all diabetic patients were included in the analysis because in the higher age groups the number of diabetic patients on oral hypoglycaemic drugs or without medication was very high. Stratification of patients into 10-year age groups revealed an increase in the prevalence of diabetes in higher age groups. However, many patients whose illness had presumably lasted less than 10 years were included. Costs have been shown to increase considerably only $8-10$ years after diagnosis of diabetes [17, 23].

A comparison of studies of the indirect costs of diabetes in Germany is difficult because different approaches have been used in the different studies. In the CODE-2 study, the indirect costs of early retirement were calculated as costs for social security and did not appear in the records of health insurance companies [3]. In the CoDiM study, inability to work and early retirement were calculated as national economic costs (according to the human capital theory). These indirect costs were approximately half (48\%) of the costs of the components included in the analysis of the CoDiM study. This percentage falls within the range for other cost-of-health studies in the USA, Canada and Sweden (25-64\%) [2].

\section{Advantages and limitations of the study}

Data used in the CoDiM study were collected primarily for administrative purposes. In comparison to information collected by means of field research, these data are costbased, contemporary, without survey or selection bias, and based on longer periods of observation. The data were derived from electronically stored data from a random sample of insured persons with membership in AOK Hesse. The study encompassed all rendered medical care services. None of the following was subjected to selection 
or recall bias: medical facilities attended by the patient, clinicians or services delegated by the clinician. The study involved an insurance-based sample rather than a clinicianbased sample, as used in some of the CODE-2 study arms. In addition, previous survey-based or field studies have had lower patient participation and completeness of data was not guaranteed.

The strength of the AOK database lies in the comprehensive and detailed description of costs related to diabetes care. All costs reimbursed by the insurance fund were included but there are obvious limitations concerning the estimation of indirect cost. Only two cost components were estimated on the basis of individual data available in the AOK database and information based on aggregated data from the Federal Statistical Office (sex-specific average income). Other elements of indirect costs (i.e. premature mortality, occupational retraining) are not available in the AOK database.

The diabetic cohort was identified on the basis of diagnosis but this method could not be externally validated because of data protection requirements. However, comparison of data from an earlier study, which used similar methods to identify the diabetic cohort from AOK administrative data, with data from the former East German diabetes registry, revealed an almost identical prevalence [25]. Hence, it is likely that the method applied provided a good estimation of the actual prevalence of this welldefined disease.

As a prevalence-based cost-of-illness study, the CoDiM study included all cases of diabetes in the specified year, regardless of whether they were diagnosed before or during that year. Diabetes is a chronic illness so patients included in the study within one year will be at different stages of illness. Different severities of diabetes are associated with different costs [17]. Costs within the first year of diagnosis are generally high because of the extensive clinical investigations required; costs then decrease, but in the following years increase once more, so that 7 years after diagnosis the costs are well above initial costs. The costs per patient calculated in this study for the year 2001 represent a cross-section of patients in various stages of diabetes.

The insured sample was derived from an insurance company (AOK) from a single region of Germany (Hesse). The population of the state of Hesse was selected because it is generally comparable to the German population. With respect to the members of $\mathrm{AOK}$, there is a difference to the German population in the distribution of age. Therefore, data were standardised according to the age and sex distribution of the German population.

In conclusion, this study provides a comprehensive cost analysis for a large representative sample of patients with diabetes using a 'bottom-up' approach. The results indicate that both age and type of treatment are strong determinants of costs. The high excess costs were largely the result of the treatment of complications. As the prevalence of diabetes in Germany and in other European countries continues to grow in the years to come, as a result of improved medical treatment and the increasing prevalence of the diabetes risk factor of obesity, diabetes will continue to be a heavy burden on health budgets. As seen in this study, excess costs increase with age in severely ill, insulin-treated patients; therefore, the importance of preventing the complications of diabetes through use of more effective treatment regimens is obvious.

Acknowledgements The authors would like to thank AOK Hesse and KV Hesse for contributing data for the 'insured sample AOK Hessen/KV Hessen'. We also thank E. Huppertz (Sanofi Aventis Germany) and E. Stridde (Pfizer Germany) for their constructive comments.

Duality of Interest The study was financially supported by the German Diabetes Foundation, Aventis Pharma Germany and Pfizer Germany with unrestricted grants.

\section{References}

1. American Diabetes Association (1998) Economic consequences of diabetes mellitus in the US in 1997. Diabetes Care 21:296-309

2. Ettaro L, Songer TJ, Zhang P, Engelgau MM (2004) Cost-ofillness studies in diabetes mellitus. Pharmacoeconomics 22:149-164

3. Liebl A, Neiß A, Spannheimer A, Reitberger U, Wagner T, Görtz A (2001) Costs of type 2 diabetes in Germany. Results of the Code-2 study. Dtsch Med Wochenschr 126:585-589

4. Jönsson B (2002) Revealing the cost of type II diabetes in Europe. Diabetologia 45:5-12

5. Ricordeau P, Weill A, Vallier N et al (2003) The prevalence and cost of diabetes in metropolitan France: what trends between 1998 and 2000? Diabetes Metab 29:497-504

6. Brandle M, Zhou H, Smith BRK et al (2003) The direct medical costs of type 2 diabetes. Diabetes Care 26:2300-2304

7. Henriksson F, Agardh CD, Berne C, Bolinder J, Jonnson B (1999) Health economics analysis of diabetes is necessary. It facilitates decision-making and international comparison. Lakartidningen 96:3915-3916, 3919

8. Redekop WK, Koopmanschap MA, Rutten GEHM, Wolffenbuttelt BHR, Stolk RP, Niessen LW (2002) Resource consumption and costs in Dutch patients with type 2 diabetes mellitus: results from 29 general practices. Diabet Med 19:246-253

9. Selby JV, Ray GT, Zhang D, Colby CJ (1997) Excess costs of medical care for patients with diabetes in a managed care population. Diabetes Care 20:1396-1402

10. Hauner H, Köster I, von Ferber L (2003) Prevalence of diabetes mellitus in Germany 1998-2001. Dtsch Med Wochenschr 128:2632-2638

11. Social Security Code (2004). 31st edition. Deutscher Taschenbuch Verlag, München

12. National Association of Statutory Health Insurance Physicians (KBV) (ed) (2001) Uniform Value Scale (EBM), Date 1 October 2001. Deutscher Ärzte-Verlag, Ulm

13. Federal Statistical Office (ed) (2003) Wages and salaries in Germany. Content and wage structure collection 2001. Wiesbaden, p 14

14. German Federal Statistical Office: Total health expenditures according to OECD criteria. Available from: http://www. destatis.de/basis/d/ges/gesutab4.htm. Accessed 5 April 2004

15. Liebl A, Spannheimer A, Reitberger U, Görtz A (2002) Costs of late complications in type 2 diabetes mellitus in Germany: results of the Code-2 study. Med Klin 97:713-719

16. American Diabetes Association (2003) Economic costs of diabetes in the US in 2002. Diabetes Care 26:917-932 
17. Brown JB, Nichols GA, Glauber HS, Bakst AW (1999) Type 2 diabetes: incremental medical care costs during the first 8 years after diagnosis. Diabetes Care 22:1116-1124

18. Schölkopf M, Stapf-Fine H (2004) Medical hospital care in international comparison. A review with conclusions for the German discussion on reforms. J Public Health 12:185-198

19. Rubin RJ, Altman WM, Mendelson DN (1994) Health care expenses for people with diabetes mellitus. J Clin Endocrinol Metab 78:809A-F

20. Jacobs P, Blanchard JF, James RC, Depew N (2000) Excess costs of diabetes on aboriginal population of Manitoba, Canada. Can J Public Health 91:298-301

21. Jonnson PM, Marke L, Nystrom L, Wall S, Ostman J (2000) Excess costs of medical care 1 and 8 years after diagnosis of diabetes: estimates from young and middle aged incidence cohort in Sweden. Diabetes Res Clin Pract 50:35-47
22. Ramsey S, Summers KH, Leong SA, Birnbaum HG, Kemner JE, Greenberg P (2002) Productivity and medical costs of diabetes in a large employer population. Diabetes Care 25:23-29

23. Henriksson F, Agardh CD, Berne C et al (2000) Direct medical costs for patients with type 2 diabetes in Sweden. J Intern Med 248:387-396

24. Brown JB, Nichols GA, Glauber HS, Bakst AW, Schaeffer M, Kelleher CC (2001) Health care costs associated with escalation of drug treatment in type 2 diabetes mellitus. Am J Health-Syst Pharm 58:151-157

25. von Ferber L, Salzsieder E, Hauner H et al (1993) Diabetes prevalence from health insurance data: evaluation of estimates by comparison with a population-based diabetes register Diabetes Metab 19:89-95 